\title{
Guest Editorial: Special Cluster on Microwave Medical Imaging
}

T HE CONCEPT of using of nonionizing microwave-frequency electromagnetic waves to image the human body has intrigued engineers and clinicians alike for several decades. Low-power microwaves can be used to sense the dielectric properties of human tissue in a low-cost manner that does not pose health risks to the patient. Yet numerous challenges need to be overcome in order to achieve the promise of microwave imaging in a clinical setting. The letters in this Special Cluster explore solutions to a variety of these challenges.

Throughout this editorial, our use of the term "microwave imaging" denotes an active approach wherein microwave energy is transmitted into tissue. This is in contrast to passive microwave imaging, known as radiometry or thermography (e.g., [1]), wherein microwave emissions due to blackbody radiation are detected and used to measure temperature differences in tissue.

\section{A. Physical Basis for Microwave Medical Imaging: Tissue Dielectric Properties}

Dielectric properties of human tissues have been assessed to further our basic understanding of the interactions between electromagnetic fields and tissues and to provide a foundation for applications ranging from dosimetry to diagnosis and treatment. Foster and Schwan provide a historical perspective on work in this field [2], while Gabriel et al. provide both a summary of reported measurements for a variety of tissues [3], as well as extensive measurement data for numerous healthy tissues [4].

At microwave frequencies, the reported properties of tissues suggest the feasibility of imaging, as well as challenges related to this task. First, different tissues have different properties that are typically related to the water content. While this suggests that tissues may be differentiated with microwaves, detection of disease or anomalies relies on differences between properties of healthy and diseased tissues or biological materials. These differences may be small, resulting in significant dynamic range requirements for practical systems. Detection may be further complicated by the presence of multiple tissues with different properties, resulting in complex scattering scenarios. Second, the properties of tissues change with frequency. Thus, while spectroscopy may have a role to play in imaging and sensing, frequency variation introduces additional unknown parameters, as well as distortion of wideband signals during propagation through tissues. Third, conductivity of tissues typically increases with frequency, resulting in a tradeoff between resolution and depth of penetration, as well as adding to the aforementioned dynamic range challenges. This complex set of challenges implies that microwave imaging and sensing

Digital Object Identifier 10.1109/LAWP.2013.2240569 may find greater success with particular tissues or biological materials where significant differences in healthy and diseased states exist. In addition, tissues that are more readily accessible by sensors reduce both dynamic range requirements and the complexity of the imaging problem. To this end, researchers have investigated the properties of healthy and diseased tissues, including liver [5], skin [6], bone [7], and heart [8].

Of particular interest to this Special Cluster are reports of the properties of healthy and malignant breast tissues. Microwave imaging of the breast is a promising application due to the low attenuation expected in fatty tissues, as well as the accessibility of the breast for imaging. The dielectric properties of breast tissue were first investigated over 60 years ago [9]. A relatively recent large-scale study of excised tissues reported the properties of healthy breast tissues with particular attention paid to the composition of the samples (i.e., percentage of fat, glandular, connective tissues) [10]. A companion study revealed that the properties of malignant tissue differ substantially from fatty tissue, but exhibit reduced contrast when compared to healthy glandular tissue [11]. These studies demonstrate both the potential for microwave breast imaging, as well as the challenge of detecting and localizing small changes in complex tissue structures. To enhance contrast between healthy and diseased tissues, recent work has suggested altering the microwave frequency properties of breast tumors via contrast agents [12]-[14].

\section{B. Microwave Medical Imaging: Past and Present}

Microwave medical imaging has been the focus of significant research effort in the electromagnetics community in the 21 st century. However, it is not a new research topic. While the field of active microwave imaging is by no means as old as that of radiography, for which the first "medical" X-ray dates back to 1895 [15], it does have a rich history of pioneering research in the later half of the 20th century.

In the 1980's, algorithm development for microwave tomography resulted in advances in both qualitative and quantitative reconstructions. Qualitative imaging algorithms (e.g., [20]) made use of the linear relationship between induced currents and measured scattered fields to permit retrieval of the current profile with a computational efficiency that was sufficient for quasi-real-time imaging. An early prototype used to test these algorithms was the $2.45-\mathrm{GHz}$ microwave "camera" [21]. Quantitative imaging algorithms (e.g., [22]) made use of linear scattering approximations to permit dielectric properties retrieval. However, for high-contrast scenarios relevant to medical imaging, the accuracy of these algorithms was severely degraded.

Advances in computing power in the 1990's made nonlinear optimization techniques feasible and led to the emergence of iterative techniques such as the Born iterative method and distorted Born iterative method for quantitative imaging [23]. 
The latter is equivalent to Gauss-Newton or Newton-Kantorovich methods of nonlinear optimization [24]. These iterative techniques are computationally expensive and require regularization to overcome the ill-posedness, but they offer improved accuracy for high-contrast scenarios. During this time period, a variety of laboratory prototypes were developed, and microwave imaging experiments were reported for tissue-equivalent phantoms as well as actual tissue samples, such as a perfused canine heart [25]. By the end of the 20th century, a clinical prototype for 2-D microwave breast tomography had been demonstrated [26]. Further advances over the past decade have included a large-scale clinical study of 2-D microwave breast imaging [27], [28], microwave breast imaging studies using "virtual patients" (MRI-derived numerical phantoms) in 2-D (e.g., [29]-[32]) and 3-D (e.g., [33] and [34]), and preliminary clinical images obtained with a 3-D microwave breast imaging system [35].

The late 1990s also brought about the proposal of an entirely different class of techniques, namely radar-based imaging methods, for microwave detection of breast tumors [36]-[38]. Such techniques seek to spatially focus the received scattered signals to locate strong scatterers. After the publication of initial studies of pulsed "confocal microwave imaging" in 2001 and 2002 [39], [40], interest in radar-based methods grew substantially through the first decade of the 21 st century, fueled in part by an incomplete understanding of the dielectric properties of breast tissue, which led to the assumption that tumors are strong scattering objects in a background environment that generates a relatively weak level of clutter. Imaging algorithms for this class of techniques include various flavors of beamforming (e.g., [41]-[45]) as well as time-reversal techniques (e.g., [46]). Since these radar techniques require the transmission and reception of ultrawideband (UWB) signals, significant effort over the past decade has also been devoted to the design of suitable UWB antennas and arrays (e.g., [47]-[50]). Preliminary clinical data has also been obtained with radar-based systems designed for breast cancer detection [51], [52].

In all of the inverse scattering (tomographic) and radar-based methods described above, microwave energy is transmitted into the tissue, and the scattered microwave signals are measured. Over the past 15 years, an alternative hybrid approach known as microwave-induced thermoacoustic tomography (MI-TAT) has been explored (e.g., [53]-[55]). In MI-TAT, externally applied microwaves induce selective heating of higher conductivity tissues, such as tumors. The resulting thermoelastic expansion of tumors generates acoustic signals that are measured and processed to form an image with ultrasound resolution but based on dielectric contrast.

\section{Open Challenges and Future Trends}

Notwithstanding the advancements witnessed over the last four decades, the application of microwaves in medical imaging remains a challenging field with several open issues related to hardware development and prototyping, methods of testing and validation, and development of algorithms able to reliably and efficiently deal with the complexity of inversion problems. Indeed, the use of microwave techniques to image isolated regions (e.g., brain, bones, and breast) as well as full human bodies poses unique methodological and technological challenges, which are expected to spur significant research efforts over the next years.

1) Experimental/System Level: From the experimental viewpoint, the capability to efficiently couple the microwave power into biological tissues still represents an important issue in practical imaging systems. To overcome the limitations of losses introduced by traditional coupling liquids as well as their associated logistical challenges, innovative antennas to be placed in close proximity or in contact with the tissues to be imaged are being investigated (e.g., [56]). Toward this end, the main design constraints reside in the size, the shape, the efficiency, and the bandwidth of the antenna, which are particularly challenging because of the inherent variability of the dielectric properties of biological tissues. As a consequence, future efforts devoted to the design of compact, high-performance antennas for microwave imaging systems are envisaged.

Another current significant challenge in full-scale microwave imaging system deployment arises from the necessity to achieve a very large dynamic range (DR) in order to reliably acquire the weak scattered field caused by the dielectric discontinuities (e.g., pathologies) to be imaged. Indeed, DR requirements as high as $100 \mathrm{~dB}$ are not unusual for microwave imaging purposes, but they are often not achieved by commercial network analyzers in practical settings [57]. Technological solutions to enhance the retrieval performance are expected to include low noise amplifiers, custom-built receivers, suitable channel-to-channel isolation strategies, and clutter suppression techniques. More accurate calibration procedures will also contribute to enhancing the sensitivity of microwave imaging systems (thus reducing the DR requirements), and they will likely continue to garner attention in the future (e.g., [58]). Suitable approaches to compensate for the variability in the dielectric properties of the biological tissues and in the antenna gain and coupling (which depend on the target, when near-field sensors are considered) are also expected.

Alternatively, enhancing the contrast between the tissues to be imaged represents an interesting research path to reduce the need for large DR. In this framework, multiphysics techniques, such as those based on the coupling of microwave and acoustic/ mechanic excitations [59], represent an endogenous contrastenhancement approach to improve the sensitivity of microwave imaging systems. Work on achieving contrast enhancement exogenously through the use of innovative selective targeting approaches with nanoparticles (e.g., carbon nanotubes, or ferroelectric or magnetic nanoparticles [13], [14]) is also expected to continue. Several critical issues that remain to be addressed include increasing the achievable concentration levels within the pathological tissues, increasing the microwave response of nanoparticles through improved fabrication procedures, and establishing their biocompatibility (which is currently guaranteed only for some specific agents).

A well-known limitation of microwave imaging systems is also represented by the tradeoff between "achievable resolution" and "penetration depth." In this framework, multifrequency/UWB imaging systems as well as super-resolution reconstruction strategies (e.g., [60] and [61]) are expected to 
be further developed over the next years to address such a fundamental issue.

2) Modeling/Methodological Level: Accurate numerical modeling of biological structures and acquisition systems represents another key challenge in the microwave medical imaging research. While anatomically realistic numerical breast phantoms for 3-D modeling are readily available [62], [63], their exploitation in conjunction with full 3-D system models, including the actual antennas, measurement tank, and feeding network, still presents challenges from a computational viewpoint. The development of innovative numerical modeling strategies is expected to play a key role in the development of microwave medical imaging technologies.

Several challenges will also have to be overcome from the methodological point of view. The difficulties of the inverse problems associated with microwave medical imaging, namely their nonlinearity and ill-posedness/ill-conditioning [64], as well as the required size/resolution have made the development of robust, accurate, and fast imaging algorithms an extremely challenging task. The introduction of a priori information concerning the type, shape, and/or dielectric properties of the biological tissues under investigation (e.g., [33], [65], and [66]) currently seems to be one of the most promising research areas to increase the reliability, speed, and robustness of inversion methodologies. The development of algorithms able to effectively exploit parallel computing architectures or GPUs within the inversion process (e.g., [33]), as well as to adaptively allocate the problem unknowns within the investigation domain (e.g., through enhanced multiresolution/multigrid strategies) is also envisaged.

\section{Scanning the Issue}

The Special Cluster includes 10 letters representing the state-of-the-art work being carried out in more than 14 institutes around the world in six countries. This collection provides a comprehensive overview of some the most interesting advancements in microwave medical imaging from the algorithmic, modeling, and experimental viewpoint, and it highlights the potential and some of the future trends of these methods and technologies. The letters address a wide variety of problems including well-established issues, such as modeling and fabrication of 2-D/3-D microwave imaging systems, as well as more recent application domains, such as contrast enhancement techniques.

The dielectric characterization of saline-based solutions relevant to medical treatment is the topic addressed in the letter by Jensen $e t$ al., while the letters by Laurin $e t$ al. and by LoVetri et al. are devoted to the development and experimental testing of new microwave imaging algorithms using realistic phantoms and/or patients. In the same framework, the first letter by Burfeindt et al. proposes 3-D-printed breast phantoms for preclinical experiments, while the development, fabrication, and measurement of innovative UWB sensors for microwave imaging is addressed in the letter by Bourqui et al.

Innovative numerical approaches to reliably simulate complex imaging systems are proposed in the letter by Attardo et al., while the letter by Guardiola et al. and the second letter by Burfeindt et al. address the development of new imaging approaches and their validation against simulated array measurements obtained from realistic numerical phantoms. Finally, innovative imaging modalities that enhance the sensitivity of breast cancer detection (through magnetic nanoparticle contrast agents) and imaging resolution (through a thermoacoustic approach) are discussed in the letter by Bucci et al. and by Wang et al., respectively

To conclude this guest editorial, we would like to thank the Editor-in-Chief, Prof. Gianluca Lazzi, for the opportunity to serve as Guest Editors of this Special Cluster, as well as for his continuous support and tremendous help, which made our efforts easier. This Special Cluster could not have been possible without the generous support of over 40 reviewers. We would like express our sincere gratitude to all of the authors for their patience with us and with the review process. Both those authors whose letters appear here and those whose contributions did not make it through the revision process by the deadline spent considerable efforts on their manuscripts and should be congratulated.

We hope that you will find this Special Cluster interesting and thought-provoking on the subject of microwave imaging. The work in these letters is proof that this topic continues to thrive as a vital interdisciplinary research field with significant scientific and industrial opportunities.

\section{SusAn C. HAgness, Guest Editor University of Wisconsin-Madison Madison, WI 53706 USA (e-mail: hagness@engr.wisc.edu)}

\section{ELISE C. FeAR, Guest Editor University of Calgary Calgary, AB T2N 1N4 Canada}

ANDREA MASSA, Guest Editor
University of Trento
Trento, 38123 Italy

\section{REFERENCES}

[1] H. Ohba, K. Abe, S. Mizushina, S Mizoshiri, and T. Sugiura, "Recent trends in medical microwave radiometry," IEIC Trans. Commun. E, vol. 78-B, pp. 789-798, 1995.

[2] K. R. Foster and H. P. Schwan, "Dielectric properties of tissues and biological materials: a critical review," CRC Crit. Rev. Biomed. Eng., vol. 17, no. 1, pp. 25-104, 1989.

[3] C. Gabriel, S. Gabriel, and E. Corthout, "The dielectric properties of biological tissues. I. Literature survey," Phys. Med. Biol., vol. 41, no. 11, pp. 2231-2249, 1996.

[4] S. Gabriel, R. W. Lau, and C. Gabriel, "The dielectric properties of biological tissues. III. Parametric models for the dielectric spectrum of tissues," Phys. Med. Biol., vol. 41, no. 11, pp. 2271-2293, 1996.

[5] A. P. O'Rourke, M. Lazebnik, J .M. Bertram, M .C. Converse, S. C. Hagness, J. G. Webster, and D. M. Mahvi, "Dielectric properties of human normal, malignant and cirrhotic liver tissue: in vivo and ex vivo measurements from 0.5 to $20 \mathrm{GHz}$ using a precision open-ended coaxial probe," Phys. Med. Biol., vol. 52, no. 15, pp. 4707-4719, 2007.

[6] T. Sunaga, H. Ikehira, S. Furukawa, H. Shinkai, H. Kobayashi, Y. Matsumoto, T. Obata, S. Tanada, H. Murata, and Y. Sasaki, "Measurement of the electrical properties of human skin and the variation among subjects with certain skin conditions," Phys. Med. Biol., vol. 47, no. 1, pp. $11-15,2002$.

[7] P. M Meaney, T. Zhou, D. Goodwin, A. Golnabi, E. Attardo, and K. Paulsen, "Bone dielectric property variation as a function of mineralization at microwave frequencies,," Int. J. Biomed. Imag, vol. 2012, p. 649612,2012 
[8] S. Y. Semenov, R. H. Svenson, V. G. Posukh, A. Nazarov, Y. Sizov, A. Bulyshev, A. Souvorov, W. Chen, J. Kasell, and G. Tatsis, "Dielectrical spectroscopy of canine myocardium during acute ischemia and hypoxia at frequency spectrum from $100 \mathrm{kHz}$ to $6 \mathrm{GHz}$," IEEE Trans. Med. Imag., vol. 21, no. 6, pp. 703-707, Jun. 2002.

[9] T. S. England and N. A. Sharples, "Dielectric properties of the human body in the microwave region of the spectrum," Nature, vol. 163, pp. 487-488, Mar. 1949.

[10] M. Lazebnik, L. McCartney, D. Popovic, C. B. Watkins, M. J. Lindstrom, J. Harter, S. Sewall, A. Magliocco, J. H. Booske, M. Okoniewski, and S. C. Hagness, "A large-scale study of the ultrawideband microwave dielectric properties of normal breast tissue obtained from reduction surgeries," Phys. Med. Biol., vol. 52, no. 10, pp. 2637-2656, May 21, 2007.

[11] M. Lazebnik, D. Popovic, L. McCartney, C. B. Watkins, M. J. Lindstrom, J. Harter, S. Sewall, T. Ogilvie, A. Magliocco, T. M. Breslin, W. Temple, D. Mew, J. H. Booske, M. Okoniewski, and S. C. Hagness, "A large-scale study of the ultrawideband microwave dielectric properties of normal, benign and malignant breast tissues obtained from cancer surgeries," Phys. Med. Biol., vol. 52, no. 20, pp. 6093-6115, Oct. 21, 2007.

[12] A. Mashal, J. H. Booske, and S. C. Hagness, "Toward contrast-enhanced microwave-induced thermoacoustic imaging of breast cancer: an experimental study of the effects of microbubbles on simple thermoacoustic targets," Phys. Med. Biol., vol. 54, no. 3, pp. 641-650, Feb. 7, 2009.

[13] A. Mashal, B. Sitharaman, X. Li, P. Avti, A. V. Sahakian, J. H. Booske, and S. C. Hagness, "Toward carbon-nanotube-based theranostic agents for microwave detection and treatment of breast cancer: Enhanced dielectric and heating response of tissue-mimicking materials," IEEE Trans. Biomed. Eng., vol. 57, no. 8, pp. 1831-1834, Aug. 2010.

[14] G. Bellizzi, O. M. Bucci, and I. Catapano, "Microwave cancer imaging exploiting magnetic nanoparticles as contrast agent," IEEE Trans. Biomed. Eng., vol. 58, no. 9, pp. 2528-2536, Sep. 2011.

[15] National Library of Medicine, Bethesda, MD, "U.S. National Library of Medicine dream anatomy gallery," Accessed Jan. 9, 2013 [Online]. Available: http://www.nlm.nih.gov/dreamanatomy/da_g_Z-1.html

[16] L. E. Larsen and J. H. Jacobi, "Microwave interrogation of dielectric targets. Part I: By scattering parameters," Med. Phys., vol. 5, no. 6, pp. $500-508,1978$.

[17] J. H. Jacobi and L. E. Larsen, "Microwave interrogation of dielectric targets. Part II: By microwave time delay spectroscopy," Med. Phys., vol. 5, no. 6, pp. 509-513, 1978.

[18] L. E. Larsen and J. H. Jacobi, "Microwave scattering parameter imagery of an isolated canine kidney," Med. Phys., vol. 6, no. 5, pp. 394-403, 1978.

[19] J. H. Jacobi and L. E. Larsen, "Microwave time delay spectroscopic imagery of isolated canine kidney," Med. Phys., vol. 7, no. 1, pp. 1-7, 1980.

[20] C. Pichot, L. Jofre, G. Peronnet, and J. C. Bolomey, "Active microwave imaging of inhomogeneous bodies," IEEE Trans. Antennas Propag., vol. AP-33, no. 4, pp. 416-425, Apr. 1985.

[21] J. Bolomey, A. Izadnegahdar, L. Jofre, C. Pichot, G. Peronnet, and M. Solaimani, "Microwave diffraction tomography for biomedical applications," IEEE Trans. Microw. Theory Tech., vol. MTT-30, no. 11, pp. 1998-2000, 1982.

[22] D. Ghodgaonkar and M. Hagmann, "Estimation of complex permittivities of three-dimensional inhomogeneous biological bodies," IEEE Trans. Microw. Theory Tech., vol. MTT-31, no. 6, pp. 442-446, Jun. 1983.

[23] W. C. Chew, Waves and Fields in Inhomogeneous Media. Piscataway, NJ: IEEE Press, 1995.

[24] R. F. Remis and P. M. van den Berg, "On the equivalence of the Newton-Kantorovich and Distorted Born methods," Inverse Problems, vol. 16, no. 1, pp. 1-4, 2000.

[25] S. Y. Semenov, R. H. Svenson, A. E. Bulyshev, A. E. Souvorov, A. E. Souvorov, V. Y. Borisov, Y. Sizov, A. N. Starostin, K. R. Dezern, G. P. Tatsis, and V. Y. Baranov, "Microwave tomography: Two-dimensional system for biological imaging," IEEE Trans. Biomed. Eng., vol. 43, no. 9, pp. 869-877, Sep. 1996.

[26] P. M. Meaney, M. W. Fanning, D. Li, S. P. Poplack, and K. D. Paulsen, "A clinical prototype for active microwave imaging of the breast," IEEE Trans. Microw. Theory Tech., vol. 48, no. 11, pp. 1841-1853, Nov. 2000.

[27] P. M. Meaney, M. W. Fanning, T. Raynolds, C. J. Fox, Q. Q. Fang, C. A. Kogel, S. P. Poplack, and K. D. Paulsen, "Initial clinical experience with microwave breast imaging in women with normal mammography," Acad. Radiol., vol. 14, no. 2, pp. 207-218, Feb. 2007.
[28] S. P. Poplack, T. D. Tosteson, W. A. Wells, B. W. Pogue, P. M. Meaney, A. Hartov, C. A. Kogel, S. K. Soho, J. J. Gibson, and K. D. Paulsen, "Electromagnetic breast imaging: Results of a pilot study in women with abnormal mammograms," Radiology, vol. 243, no. 2, pp. 350-359, May 2007.

[29] D. W. Winters, E. J. Bond, B. D. Van Veen, and S. C. Hagness, "Estimation of the frequency-dependent average dielectric properties of breast tissue using a time-domain inverse scattering technique," IEEE Trans. Antennas Propag., vol. 54, no. 11, pp. 3517-3528, Nov. 2006.

[30] J. E. Johnson, T. Takenaka, and T. Tanaka, "Two-dimensional time-domain inverse scattering for quantitative analysis of breast composition," IEEE Trans. Biomed. Eng., vol. 55, no. 8, pp. 1941-1945, Aug. 2008.

[31] C. Gilmore, A. Abubakar, W. Hu, T. Habashy, and P. van den Berg, "Microwave biomedical data inversion using the finite-difference contrast source inversion method," IEEE Trans. Antennas Propag., vol. 57, no. 5, pp. 1528-1538, May 2009.

[32] N. Irishina, D. Alvarez, O. Dorn, and M. Moscoso, "Structural level set inversion for microwave breast screening," Inverse Problems, vol. 26, no. 3, p. 035015 , Mar. 2010.

[33] J. D. Shea, P. Kosmas, S. C. Hagness, and B. D. Van Veen, "Three-dimensional microwave imaging of realistic numerical breast phantoms via a multiple-frequency inverse scattering technique," Med. Phys., vol. 37, no. 8, pp. 4210-4226, Aug. 2010.

[34] J. D. Shea, P. Kosmas, B. D. Van Veen, and S. C. Hagness, "Contrastenhanced microwave imaging of breast tumors: A computational study using 3D realistic numerical phantoms," Inverse Problems, vol. 26, no. 7, p. 074009 , Jul. 2010

[35] T. M. Grzegorczyk, P. M. Meaney, P. A. Kaufman, R. M. diFlorio-Alexander, and K. D. Paulsen, "Fast 3-D tomographic microwave imaging for breast cancer detection," IEEE. Trans. Med. Imag., vol. 31, no. 8, pp. 1584-1592, Aug. 2012.

[36] S. C. Hagness, A. Taflove, and J. E. Bridges, "Two-dimensional FDTD analysis of a pulsed microwave confocal system for breast cancer detection: Fixed-focus and antenna-array sensors," IEEE Trans. Biomed. Eng., vol. 45, no. 12, pp. 1470-1479, Dec. 1998.

[37] S. C. Hagness, A. Taflove, and J. E. Bridges, "Three-dimensional FDTD analysis of a pulsed microwave confocal system for breast cancer detection: Design of an antenna-array element," IEEE Trans. Antennas Propag., vol. 47, no. 5, pp. 783-791, May 1999.

[38] E. Fear and M. Stuchly, "Microwave detection of breast cancer," IEEE Trans. Microw. Theory Tech., vol. 48, no. 11, pp. 1854-1863, Nov. 2000.

[39] X. Li and S. C. Hagness, "A confocal microwave imaging algorithm for breast cancer detection," IEEE Microw. Wireless Compon. Lett., vol. 11, no. 3, pp. 130-132, Mar. 2001.

[40] E. C. Fear, X. Li, S. C. Hagness, and and M. A. Stuchly, "Confocal microwave imaging for breast cancer detection: Localization of tumors in three dimensions," IEEE Trans. Biomed. Eng., vol. 49, no. 8, pp. 812-822, Aug. 2002.

[41] E. J. Bond, X. Li, S. C. Hagness, and B. D. Van Veen, "Microwave imaging via space-time beamforming for early detection of breast cancer," IEEE Trans. Antennas Propag., vol. 51, no. 8, pp. 1690-1705, Aug. 2003.

[42] J. M. Sill and E. C. Fear, "Tissue sensing adaptive radar for breast cancer detection - experimental investigation of simple tumor models," IEEE Trans. Microw. Theory Tech., vol. 53, no. 11, pp. 3312-3319, Nov. 2005

[43] Y. Xie, B. Guo, L. Xu, J. Li, and P. Stoica, "Multistatic adaptive microwave imaging for early breast cancer detection," IEEE Trans. Biomed. Eng., vol. 53, no. 8, pp. 1647-1657, Aug. 2006.

[44] M. Klemm, I. J. Craddock, J. A. Leendertz, A. Preece, and R. Benjamin, "Improved Delay-and-Sum Beamforming Algorithm for Breast Cancer Detection," Int. J. Antennas Propag., vol. 2008, p. 761402, 2008.

[45] D. Byrne, M. O'Halloran, M. Glavin, and E. Jones, "Data independent radar beamforming algorithms for breast cancer detection," Prog. Electromagn. Res., vol. 107, pp. 331-348, 2010.

[46] P. Kosmas and C. Rappaport, "A matched filter FDTD-based time reversal algorithm for microwave breast cancer detection," IEEE Trans. Antennas Propag., vol. 54, no. 4, pp. 1257-1264, Apr. 2006.

[47] X. Li, S. K. Davis, S. C. Hagness, D. van der Weide, and B. D. Van Veen, "Microwave imaging via space-time beamforming: Experimental investigation of tumor detection in multi-layer breast phantoms," IEEE Trans. Microw. Theory Tech., vol. 52, no. 8, pp. 1856-1865, Aug. 2004.

[48] D. A. Woten and M. El-Shenawee, "Broadband dual linear polarized antenna for statistical detection of breast cancer," IEEE Trans. Antennas Propag., vol. 56, no. 11, pp. 3576-3580, Nov. 2008. 
[49] J. Bourqui, M. Okoniewski, and E. C. Fear, "Balanced antipodal Vivaldi antenna with dielectric director for near-field microwave imaging," IEEE Trans. Antennas Propag., vol. 58, no. 7, pp. 2318-2326, Jul. 2010.

[50] D. Gibbins, M. Klemm, I. J. Craddock, J. A. Leendertz, A. Preece, and R. Benjamin, "A comparison of a wide-slot and a stacked patch antenna for the purpose of breast cancer detection," IEEE Trans. Antennas Propag., vol. 58, no. 3, pp. 665-674, Mar. 2010.

[51] T. HenrikssonM. KlemmD. Gibbins, J. Leendertz, T. Horseman, A. W. Preece, R. Benjamin, and I. J. Craddock, "Clinical trials of a multistatic UWB radar for breast imaging," in Proc. Loughborough Antennas Propag. Conf., Loughborough, U.K., Nov. 2011.

[52] J. Bourqui, J. Garrett, and E. Fear, "Measurement and analysis of microwave frequency signals transmitted through the breast," Int. Jour. Biomed. Imag., vol. 2012, p. 562563, 2012.

[53] R. Kruger, K. Miller, H. Reynolds, W. Kiser, D. Reinecke, and G. Kruger, "Breast cancer in vivo: Contrast enhancement with thermoacoustic CT at $434 \mathrm{MHz}$-Feasibility study," Radiology, vol. 216, no. 1, pp. 279-283, Jul. 2000.

[54] L. Nie, D. Xing, Q. Zhou, D. Yang, and H. Guo, "Microwave-induced thermoacoustic scanning CT for high-contrast and noninvasive breast cancer imaging," Med. Phys., vol. 35, no. 9, pp. 4026-4032, Sep. 2008.

[55] M. Pramanik, G. Ku, C. Li, and L. V. Wang, "Design and evaluation of a novel breast cancer detection system combining both thermoacoustic (TA) and photoacoustic (PA) tomography," Med. Phys., vol. 35, no. 6, pp. 2218-2223, Jun. 2008.

[56] R. K. Amineh, M. Ravan, A. Trehan, and N. K. Nikolova, "Near-field microwave imaging based on aperture raster scanning with TEM horn antennas," IEEE Trans. Antennas Propag., vol. 59, no. 3, pp. 928-940, Mar. 2011.

[57] P. M. Meaney, D. Goodwin, A. H. Golnabi, Z. Tian, M. Pallone, S. D. Geimer, G. Burke, and K. D. Paulsen, "Clinical microwave tomographic imaging of the calcaneus: A first-in-human case study of two subjects," IEEE Trans. Biomed. Eng., vol. 59, no. 12, pp. 3304-3313, Dec. 2012.
[58] C. Gilmore, P. Mojabi, A. Zakaria, M. Ostadrahimi, C. Kaye, S. Noghanian, L. Shafai, S. Pistorius, and J. LoVetri, "A wideband microwave tomography system with a novel frequency selection procedure," IEEE Trans. Biomed. Eng., vol. 57, no. 4, pp. 894-904, Apr. 2010.

[59] M. Zhao, J. D. Shea, S. C. Hagness, D. W. van der Weide, B. D. Van Veen, and T. Varghese, "Numerical study of microwave scattering in breast tissue via coupled dielectric and elastic contrasts," IEEE Antennas Wireless Propag. Lett., vol. 7, pp. 247-250, 2008.

[60] M. A. Maha and M. Moghaddam, "3D nonlinear super-resolution microwave inversion technique using time-domain data," IEEE Trans. Antennas Propag., vol. 58, no. 7, pp. 2327-2336, Jul. 2010.

[61] C. Gilmore, P. Mojabi, A. Zakaria, S. Pistorius, and J. LoVetri, "On super-resolution with an experimental microwave tomography system," IEEE Antennas Wireless Propag. Lett., vol. 9, pp. 393-396, 2010.

[62] E. Zastrow, S. K. Davis, M. Lazebnik, F. Kelcz, B. D. Van Veen, and S. C. Hagness, "Development of anatomically realistic numerical breast phantoms with accurate dielectric properties for modeling microwave interactions with the human breast," IEEE Trans. Biomed. Eng., vol. 55 , no. 12 , pp. 2792-2800, Dec. 2008.

[63] University of Wisconsin Computational Electromagnetics Laboratory, Madison, WI, "UWCEM numerical breast phantom repository," Accessed Jan. 9, 2013

[Online]. Available: http://uwcem.ece.wisc.edu/MRIdatabase/

[64] P. Rocca, M. Benedetti, M. Donelli, D. Franceschini, and A. Massa, "Evolutionary optimization as applied to inverse scattering problems," Inverse Problems, vol. 25, p. 123003, Dec. 2009

[65] A. Ashtari, S. Noghanian, A. Sabouni, J. Aronsson, G. Thomas, and S. Pistorius, "Using a-priori information for regularization in breast microwave image reconstruction," IEEE Trans. Biomed. Eng., vol. 57, no. 9, pp. 2197-2208, Sep. 2010.

[66] G. Oliveri, P. Rocca, and A. Massa, "A Bayesian-compressive-sampling-based inversion for imaging sparse scatterers," IEEE Trans. Geosci. Remote Sens., vol. 49, no. 10, pp. 3993-4006, Oct. 2011.

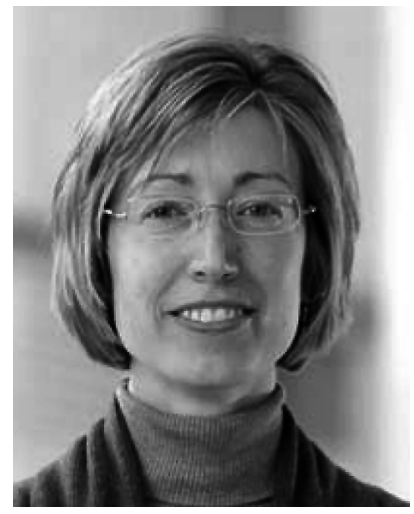

Susan C. Hagness (S'91-M'98-SM'04-F'09) received the B.S. degree with highest honors and the $\mathrm{Ph} . \mathrm{D}$. degree in electrical engineering from Northwestern University, Evanston, IL, in 1993 and 1998, respectively.

Since 1998, she has been with the Department of Electrical and Computer Engineering, University of Wisconsin-Madison, where she currently holds the title of Philip D. Reed Professor. She is also a faculty affiliate of the Department of Biomedical Engineering. Her current bioelectromagnetics research interests include microwave breast imaging, microwave thermotherapy, nanoparticles as electromagnetic theranostic agents, and computational electromagnetics theory and applications in biology and medicine.

Dr. Hagness served as an elected member of the IEEE Antennas and Propagation Society (AP-S) Administrative Committee from 2003 to 2005 and as an Associate Editor for the IEEE ANTENNAS AND WIRELESS PROPAGATION LETTERS from 2002 to 2007. She also served as Chair of Commission $\mathrm{K}$ of the United States National Committee (USNC) of the International Union of Radio Science (URSI) from 2009 to 2011, and Technical Program Chair of the 2012 IEEE International Symposium on Antennas and Propagation and USNC/URSI National Radio Science Meeting. She currently serves as Chair of the IEEE AP-S Fellows Committee. She was the recipient of the Presidential Early Career Award for Scientists and Engineers presented by the White House in 2000. In 2002, she was named one of the 100 top young innovators in science and engineering in the world by the Massachusetts Institute of Technology (MIT) Technology Review magazine. She is also the recipient of the UW-Madison Emil Steiger Distinguished Teaching Award in 2003, the IEEE Engineering in Medicine and Biology Society Early Career Achievement Award in 2004, the URSI Isaac Koga Gold Medal in 2005, the IEEE Transactions on BiOMEDiCAL ENGINEERING Outstanding Paper Award in 2007, the IEEE Education Society Mac E. Van Valkenburg Early Career Teaching Award in 2007, the UW System Alliant Energy Underkofler Excellence in Teaching Award in 2009, the Physics in Medicine and Biology Citations Prize in 2011, and the UW-Madison Kellett Mid-Career Award in 2011. 


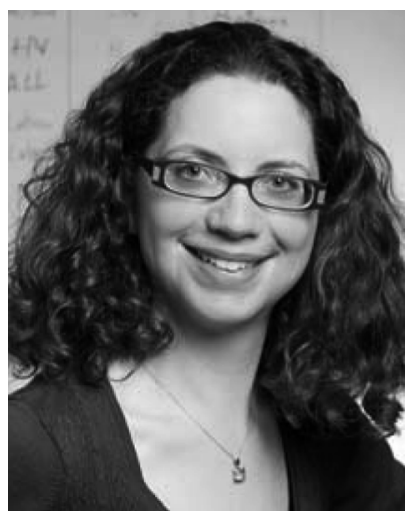

Elise C. Fear (S'98-M'02-SM'11) received the Ph.D. degree in electrical engineering from the University of Victoria, Victoria, BC, Canada, in 2001.

She joined the Department of Electrical and Computer Engineering, Schulich School of Engineering, University of Calgary, Calgary, AB, Canada, in 2001 and is currently an Associate Professor in the same department. Her research interests include microwave imaging and sensing for biomedical applications, including ultrawideband antennas and prototype system development.

Dr. Fear served as the Canadian National Committee URSI Commission K representative from 2009 to 2012. She has served an Associate Editor for the IEEE TRANSACTIONS ON BIOMEDICAL ENGINEERING since 2005, and was awarded the 2007 Outstanding Paper Award from the same journal. Her work has also been recognized with a Schulich School of Engineering Departmental Research Excellence Award in 2009, a “Top 40 under 40" from Calgary Avenue Magazine, and the University of Calgary Killam Interdisciplinary Research Prize in 2012.

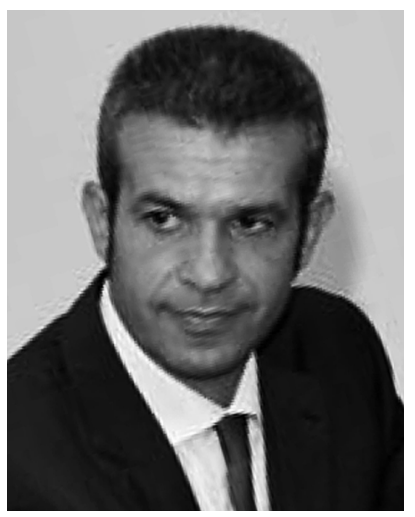

Andrea Massa (M'03) received the "Laurea" degree in electronic engineering and Ph.D. degree in electronics and computer science from the University of Genoa, Genoa, Italy, in 1992 and 1996, respectively.

From 1997 to 1999, he was an Assistant Professor of electromagnetic fields with the Department of Biophysical and Electronic Engineering, University of Genoa. From 2001 to 2004, he was an Associate Professor with the University of Trento, Trento, Italy. Since 2005, he has been a Full Professor of electromagnetic fields with the University of Trento, where he currently teaches electromagnetic fields, inverse scattering techniques, antennas and wireless communications, and optimization techniques. He is the Director of the ELEDIA Research Center, University of Trento, and Deputy Dean of the Faculty of Engineering. Moreover, he is an Adjunct Professor with Pennsylvania State University, University Park, PA, USA, and a Visiting Professor with the Missouri University of Science and Technology, Rolla, MO, USA; Nagasaki University, Nagasaki, Japan; University of Paris Sud, Orsay, France; and Kumamoto University, Kumamoto, Japan. His research work since 1992 has been principally on electromagnetic direct and inverse scattering, microwave imaging, optimization techniques, wave propagation in the presence of nonlinear media, wireless communications, and applications of electromagnetic fields to telecommunications, medicine, and biology.

Prof. Massa is a member of the PIERS Technical Committee and the Inter-University Research Center for Interactions between Electromagnetic Fields and Biological Systems (ICEmB), and he has served as the Italian representative in the general assembly of the European Microwave Association (EuMA). He currently serves as an Associate Editor of the IEEE TRANSACTIONS ON ANTENNAS AND PROPAGATION. 